\title{
Topical Intranasal Fluorescein to Diagnose and Localize Cerebrospinal Fluid Leak: A Systematic Review
}

\author{
Systematic Review (1) Hussain Albaharna ${ }^{1,3}$, (1) Mohammad Alshareef ${ }^{2,3}$, (1) Saud Alromaih ${ }^{3}$, \\ (1) Mohammad Aloulah ${ }^{3}$, (1) Saad Alsaleh ${ }^{3}$, (1) Ahmad Alroqi ${ }^{3}$ \\ ${ }^{1}$ Department of Otolaryngology-Head and Neck surgery, Qatif Central Hospital, Qatif, Eastern province, Saudi Arabia. \\ ${ }^{2}$ Department of Otolaryngology-Head and Neck Surgery, Khamis Mushait General Hospital, Khamis Mushait City, \\ Saudi Arabia \\ ${ }^{3}$ Department of Otolaryngology-Head \& Neck Surgery, King Saud University, Riyadh, Saudi Arabia.
}

\section{ORCID ID of the authors:}

H.A. 0000-0003-3345-1644;

M.A. 0000-0001-8636-6107;

Sa.A. 0000-0002-1236-2098

A.A. 0000-0002-5275-0255.

Cite this article as: Albaharna $H$, Alshareef M, Alromaih S, Aloulah M, Alsaleh S, Alrogi A. Topical Intranasal Fluorescein to Diagnose and Localize Cerebrospinal Fluid Leak: A Systematic Review. Turk Arch Otorhinolaryngol 2021; 59(3): 223-9.

Corresponding Author: Hussain Albaharna; h.albaharna@gmail.com

Received Date: 31.03 .2021 Accepted Date: 14.06 .2021

Content of this journal is licensed under a Creative Commons Attribution 4.0 International License. Available online at www.turkarchotolaryngol.net
Objective: This study evaluates the available evidence regarding using topical intranasal fluorescein (TINF) to diagnose and localize nasal cerebrospinal fluid (CSF) leak.

Methods: A literature search was conducted through PubMed, the Cochrane Database, Scopus, and Ovid to identify the articles providing insight into using TINF to diagnose CSF leak preoperatively or to localize the leak intraoperatively. The articles from the database were screened and filtered by two authors according to the selection criteria. A spreadsheet was created to collect the data including demographic characteristics, the sensitivity and specificity of TINF for diagnosing and localizing a CSF leak, the protocol of applying TINF, and the complications.

Results: After excluding duplicates and articles that did not meet our selection criteria, we included five reports in the final analysis. The average age of the 94 participants was 39.5, and there was an equal distribution of males and females. The sensitivity of TINF to make a preoperative diagnosis of CSF leak was $100 \%$, and it was $97 \%$ to localize the site intraoperatively. Complications associated with TINF were not reported in any of the reports. This review showed a grade $\mathrm{C}$ recommendation based on five case series.

Conclusion: Based on the current evidence, TINF cannot be recommended for standard clinical practice. It can, however, be considered in situations where other gold standard tools are unavailable since it is feasible and easy to use. A standardized control trial should be conducted to yield additional unbiased evidence.

Keywords: Cerebrospinal fluid rhinorrhea, paranasal sinuses, skull base, spinal puncture, transferrin, systematic review 


\section{Introduction}

Nasal cerebrospinal fluid (CSF) leak has multiple known etiologies that could be traumatic, iatrogenic, or spontaneous. In general, traumatic CSF leaks, whether accidental or iatrogenic, account for $70 \%-80 \%$ of cases (1-3). The incidence of CSF leaks range from $0.17 \%$ in endoscopic sinus surgery to around $6.0 \%$ in transsphenoidal surgery $(4,5)$, which may result in complications in about $10 \%-37 \%$ of those cases (6). Such complications include meningitis, pneumocephalus, and meningo-encephalocele (7). Management of this pathology consists of three steps. Confirming the diagnosis is the first step, in which the fluid is analyzed for beta-2 transferrin as a primary screening test in a suspected symptomatic patient (6). The reported beta- 2 transferrin sensitivity and specificity are approximately $97 \%$ and $99 \%$, respectively, with positive and negative predictive values of $97 \%$ and $99 \%$ (8). Also, beta trace protein presents in high concentration in CSF. Studies showed that the sensitivity and specificity for a beta trace protein immunoassay were $100 \%$ and $86 \%$, respectively (6). The next step is the localization of the leak site, which is necessary to repair the site in the third step. Imaging studies, including high resolution computed tomography (HRCT) of paranasal sinuses and magnetic resonance cisternography (MRC) play an essential role. Studies on HRCT in localizing a CSF leak showed sensitivity and specificity of $44 \%$ to $100 \%$ and $45 \%$ to $100 \%$, respectively $(9,10)$, while reported positive and negative predictive values were $100 \%$ and $50 \%$ to $70 \%(6,11,12)$. On the other hand, studies reported MRC accuracy, sensitivity, and specificity to be $89 \%, 87 \%$, and $100 \%$, respectively $(13,14)$. If the site of a CSF leak cannot be localized radiologically, intrathecal fluorescein (IF) can be used intraoperatively. Although IF can help with localization, it is an invasive procedure requiring lumbar puncture to administer fluorescein, increasing the operating time. Moreover, IF is not approved by the FDA. Therefore, benefits and risks should be discussed with the patient. A consent form must be signed before using IF as it may lead to complications such as malaise, headache, numbness in the extremities, seizures, and chemical meningitis (15). Published reports of implementing IF demonstrated varying rates of detecting the CSF leak site, ranging from $46 \%$ to 100\% (16-18). Therefore, topical intranasal fluorescein (TINF) has been tried $(19,20)$. This alternative technique is non-invasive, faster, and causes no complications.

We conducted a systematic review to analyze the efficacy of TINF. Using the available literature, we aimed to investigate the sensitivity, specificity, and safety of TINF. Our secondary objective was to assess the possibility of applying TINF in current practice.

\section{Methods}

\section{Data Sources and Literature Search}

We conducted an extensive literature review in different databases using specific keywords from January 2000 to December 2019:

PubMed: We used a PubMed Medical Subject Heading (MeSH) search according to the Preferred Reporting Items for Systematic Reviews and Meta-Analyses (PRISMA) guidelines with the search terms: (topical[All Fields] OR local[All Fields]) AND (intranasal[All Fields] OR ("nose"[MeSH Terms] OR "nose"[All Fields] OR "nasal"[All Fields]) AND ("fluorescein"[MeSH Terms] OR "fluorescein"[All Fields]).

Cochrane Database: We used the search terms: (topical AND nasal OR intranasal AND fluorescein).

Scopus: We used the search terms: (topical AND nasal OR intranasal AND fluorescein).

Ovid: We used the search terms: (topical AND nasal OR intranasal AND fluorescein).

Other Resources for Data: We screened the reference lists of identified publications for additional trials and contacted some authors when necessary. We also ran a non-systematic search on Google Scholar to retrieve grey literature and other sources of potential trials.

\section{Study Selection}

Following the predetermined inclusion and exclusion criteria, the studies selection was made in two stages by two authors independently. In the first stage, titles, and abstracts of the most relevant studies from the electronic database search were reviewed. The full-text articles of these retrieved studies were collected and reviewed in the second stage. In case of a discrepancy between the two reviewers, a third reviewer intervened until a consensus was reached. In cases of duplicates, the most recent version was selected.

\section{Inclusion and Exclusion Criteria}

Studies were selected if TINF was used to diagnose the presence of a CSF leak preoperatively or to localize the leak intraoperatively. To demonstrate the efficacy of TINF, this review included clinical trials, cohort studies, and diagnostic case series. Cases were included if a preoperative diagnosis of a CSF leak was made by TINF and confirmed by a beta2 transferrin test or another radiological test. If, however, a laboratory or radiological test was used to diagnose a CSF leak before applying TINF, the study was excluded. Another inclusion criterion was the identification of the leak site by 
TINF intraoperatively. If IF was used to detect the location of the leak before TINF, the study was excluded. Only studies on CSF leaks were included. However, an iatrogenic CSF leak during endoscopic skull base surgery was not counted.

\section{Data Synthesis and Data Extraction}

To ensure that data extraction was accurate and reproducible, two authors independently reviewed the studies. We created review-specific data extraction forms to make the process consistent and to avoid missing data. If additional data was needed, we sent a request with all inquiries via email to the corresponding author of the article.

For each study, we extracted the following data:

- Year of publication and country of origin

- Demographic characteristics of the patients

- Accuracy of preoperative diagnosis of CSF leak with TINF

- Accuracy of intraoperative localization using TINF

- Protocol of applying TINF

- Complications

\section{Assessment of Outcomes}

To assess the outcomes, data were divided into two categories: preoperative diagnosis and intraoperative localization. For each type of diagnosis, the sensitivity, specificity, positive prediction rate, negative prediction rate, and cause of the CSF leak were examined.

\section{Assessment of Risk of Bias in Included Studies}

The methodologies of the included studies were evaluated for bias. Since all included articles were case series, we used the Joanna Briggs Institute (JBI) critical appraisal checklist tool for case series (21).

\section{Registration}

This systematic review protocol was registered in the Prospero database with ID: CRD42020186463.

\section{Results}

\section{Results of the Search}

We found 266 articles by primary research using PubMed, the Cochrane Database, Scopus, and Ovid. After removing duplicate articles, 109 remained. The titles and abstracts were then screened by two authors independently for relevant articles based on the inclusion and exclusion criteria, and we selected eight for full-text review. Out of these, three were excluded: two had no full-text English version, and one did not meet the inclusion criteria. The five remaining articles included were all reports of case series. A flow chart of the study selection process is shown in Figure 1.

\section{Design}

All articles included in this analysis are case series. Three of them describe preoperative diagnosis and intraoperative localization of a CSF leak using TINF, while the other two report only intraoperative localization.

\section{Sample Sizes}

In total, the included case series in this review had a small sample size of 94 patients for analysis.

\section{Patient Characteristics}

Ninety-four participants were included in this review and had equal gender distribution with 47 patients in each group. The age ranged from 14-68 years with an overall mean of 39.5 years. The most common etiology of a CSF leak among patients was a spontaneous leak (55.3\%) followed by trauma (29.7\%) and iatrogenic causes (15.9\%). We counted 100 sites of CSF leaks, which exceeds the total number of cases as three patients had revision surgery, and three suffered from bilateral leaks. Patient characteristics and distribution of defect sites are shown in Table 1.

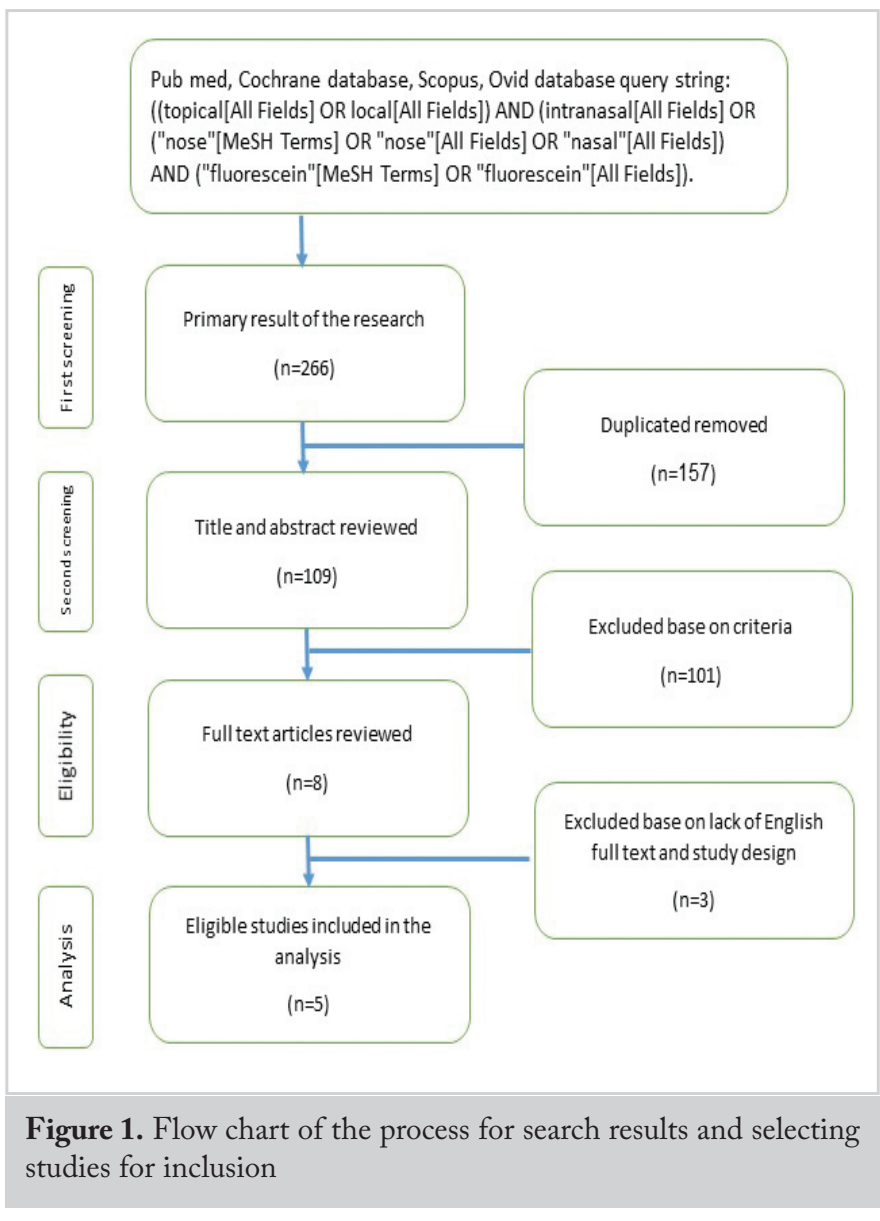


Table 1. Clinical characteristics of patients included in the review

\begin{tabular}{ll}
\hline Number of patients & $(\mathbf{n}=\mathbf{9 4})$ \\
\hline Sex (male) & $47(50 \%)$ \\
\hline Age & $(\mathrm{n}=94)$ \\
\hline Mean & 39.5 \\
\hline Cause of CSF leak & \\
\hline Spontaneous & 49 \\
\hline Traumatic & 28 \\
\hline Iatrogenic & 15 \\
\hline Meningoencephalocele & 2 \\
\hline Site of CSF leak & $\mathrm{n}=100$ \\
\hline Ethmoidal roof & 59 \\
\hline Cribriform plate & 31 \\
\hline Sphenoid & 9 \\
\hline Frontal & 1 \\
\hline CSF: Cerebrospinal fluid & \\
\hline
\end{tabular}

\section{Intervention}

There was no consistent regimen of using the fluorescein intranasally. However, all studies used plain fluorescein 5\%$10 \%$ and applied directly into the nose. A test was considered positive for the presence of CSF when the fluorescein color changed from orange to green. When we excluded the study of Jones et al. (22), which does not specify the fluorescein concentration and includes only three cases, we found 40 participants who received 5\% of fluorescein, and 51 who received $10 \%$ fluorescein. Using the chi-square test which yielded a p-value of 0.58 , we observed no significant difference between the two groups concerning the detection of CSF leak sites.

\section{Protocol for Preoperative Diagnosis}

Most authors agreed on applying fluorescein intranasally with cotton pledgets after anesthetizing the nasal mucosa. There was no agreement on the local anesthetic or decongestant used. Cotton pledgets were placed with a rigid nasal endoscope at the middle meatus, the roof of the cribriform plate, and the sphenoethmoidal recess.

\section{Protocol for Intraoperative Localization}

Various endoscopic sinus approaches were used to access the CSF leak, according to the site. Once the endoscope reached the suspected site, TINF was applied using cotton pledgets. A color change from brown to green fluorescence and sometimes streaming of the fluorescein over the nasal mucosa and blood denoted the presence CSF. If CSF was suspected to be at a specific site, the area was irrigated with saline to clean the fluorescein applied.
All authors of the included reports used the regular light source without a blue light filter to detect the fluorescein color change.

\section{Outcomes}

\section{Topical Fluorescein for Preoperative Diagnosis of CSF Leaks}

Among the included studies, only three discussed preoperative diagnosis using TINF. Sixty-four cases were tested, and a diagnosis was successfully attained in all of them with $100 \%$ sensitivity. All the included articles were case series describing cases of confirmed CSF leaks. However, there were no cohort- or case-controls to assess the specificity of TINF in rolling out CSF leaks (Table 2).

\section{Topical Fluorescein for Intraoperative Localization}

TINF was used in all 94 cases to detect the CSF leak site. Except for three cases, the leak's location was successfully identified intraoperatively, achieving a sensitivity of $97 \%$. It is unclear from the reports whether the leak sites were not visible without fluorescein supplementation and were found with fluorescein support intraoperatively. The exceptions were the three cases presented by Jones et al. (22), where the leak sites were identified after application of TINF. Specificity was not calculated since the case would not have been taken for intraoperative localization and repair without confirming the diagnosis at a prior stage (Table 2).

\section{Complications}

No complications were reported with TINF application. Thus, according to our review, using plain fluorescein 5\%$10 \%$ intranasally to detect CSF leak was safe.

\section{Quality Assessment and Level of Evidence}

Using the JBI assessment tool for case series, we checked the included articles for bias. They all generally shared the same level of bias: high selection bias on including patients of high clinical suspicion for CSF rhinorrhea, low bias in measurement outcome, low bias of result reporting, and low bias in missing data.

According to this review based on five case series describing the use of TINF (Level 4 evidence), the aggregate grade of recommendation is $\mathrm{C}$.

\section{Meta-Analysis}

We could not proceed to meta-analysis because of the lack of a standardized cohort and randomization.

\section{Discussion}

The available and commonly used methods for diagnosing a CSF leak have some limitations. Some tests, such as glucose testing, are unreliable $(23,24)$. Both beta trace protein 
and beta- 2 transferrin tests need to be performed in a specialized laboratory, which may not be always feasible (6). Although these might be inexpensive (approximately $\$ 20$ and $\$ 37.9$ for beta trace protein and beta- 2 transferrin, respectively), the samples need to be sent to tertiary labs for analysis and sometimes across borders. Days to weeks may pass before results come in. Unlike the proteins, radiological tools are expensive, ranging from $\$ 280$ for HRCT to $\$ 807$ for MRC (6). On the other hand, an ampule of $10 \%$ fluorescein costs only $\$ 1.7$ (25), and is widely available, as it is used in many ophthalmic procedures and brain tumor surgeries (26).

In areas with limited resources, alternative methods to diagnose CSF leaks have been used. These methods, such as skull X-rays or Valsalva and squatting maneuvers, are not standardized and thus have unknown efficacy rates $(27,28)$. Our review shows that TINF offers a feasible method and can be applied quickly and easily. Moreover, TINF is the only suggestive tool available for both preoperative diagnosis and intraoperative localization of CSF leaks. Although it does not substitute the gold standard tools, it should be considered in situations where such tools are not available.

Intraoperative localization of CSF leak is not straightforward. The most important advantages of using TINF over IF for intraoperative localization are safety and rapid applicability. Nonetheless, IF is a more reliable and preferable method based on its high sensitivity and specificity rate. It remains however, an invasive technique as it requires lumbar puncture, and is time-consuming and less safe than TINF, and causes discomfort to the patient (18).

One of the limitations of our review is that only case series were included. Case series can increase the evidence level, strength, and credibility of a review. However, these advantages must be balanced against the risk of bias associated with the lack of a control group. Another drawback is that none of the case series studied the efficacy of TINF in ruling out a diagnosis of CSF. Hence, the specificity of the test was not calculated in this review.

Therefore, TINF has an insufficient grade of evidence for it to be recommended in current clinical practice. More control trials would help achieve a higher level of evidence for TINF implementation.

\section{Conclusion}

This review explores the application of TINF to detect CSF leaks. The available evidence showed a low level of recommendation. To increase the amount of 
unbiased evidence, thereby provide more legitimate support, standardized control trials should be conducted.

Ethics Committee Approval: This systematic review protocol was registered in Prospero data base with ID: CRD42020186463.

Informed Consent: Not applicable since the study conducted through the databases PubMed, the Cochrane Database, Scopus, and Ovid.

Peer-review: Externally peer-reviewed.

Conflict of Interest: No conflict of interest was declared by the authors.

Financial Disclosure: The authors declared that this study received no financial support.

\section{Authorship Contributions}

Conception: H.A., S.A., Mo.A., Sa.A., A.A., Design: H.A., M.A., Sa.A., A.A., Supervision: Sa.A., A.A., Data Collection and/or Processing: H.A., M.A., Analysis and/ or Interpretation: H.A., M.A., S.A., Mo.A, Sa.A., A.A., Literature Review: H.A., M.A., Writing: H.A., M.A., Critical Review: S.A., Mo.A., Sa.A., A.A.

\section{Main Points}

- TINF is a test used to diagnose and localize CSF leak.

- Fluorescein 5\%-10\% is applied directly into the nose.

- The test result is considered positive when fluorescein color changes from orange to green.

- There is insufficient evidence to recommend using TINF in current clinical practice.

- TINF can be considered when other gold standard tools are not available.

\section{References}

1. Razeghinejad MR, Myers JS. Contemporary approach to the diagnosis and management of primary angle-closure disease. Surv Ophthalmol 2018; 63: 754-68. [Crossref]

2. Kerr JT, Chu FW, Bayles SW. Cerebrospinal fluid rhinorrhea: diagnosis and management. Otolaryngol Clin North Am 2005; 38: 597-611. [Crossref]

3. Abuabara A. Cerebrospinal fluid rhinorrhoea: diagnosis and management. Med Oral Patol Oral Cir Bucal 2007; 12: E397-400. [Crossref]

4. Shiley SG, Limonadi F, Delashaw JB, Barnwell SL, Andersen $\mathrm{PE}$, Hwang PH, et al. Incidence, etiology, and management of cerebrospinal fluid leaks following trans-sphenoidal surgery. Laryngoscope 2003; 113: 1283-8. [Crossref]
5. Ramakrishnan VR, Kingdom TT, Nayak JV., Hwang PH, Orlandi RR. Nationwide incidence of major complications in endoscopic sinus surgery. Int Forum Allergy Rhinol 2012; 2: 34-9. [Crossref]

6. Oakley GM, Alt JA, Schlosser RJ, Harvey RJ, Orlandi RR. Diagnosis of cerebrospinal fluid rhinorrhea: an evidence-based review with recommendations. Int Forum Allergy Rhinol. 2016; 6: 8-16. [Crossref]

7. Hao SP. Transnasal endoscopic repair of cerebrospinal fluid rhinorrhea: an interposition technique. Laryngoscope 1996; 106: 501-3. [Crossref]

8. Warnecke A, Averbeck T, Wurster U, Harmening M, Lenarz T, Stöver T. Diagnostic relevance of beta2-transferrin for the detection of cerebrospinal fluid fistulas. Arch Otolaryngol Head Neck Surg 2004; 130: 1178-84. [Crossref]

9. Tahir MZ, Khan MB, Bashir MU, Akhtar S, Bari E. Cerebrospinal fluid rhinorrhea: an institutional perspective from Pakistan. Surg Neurol Int 2011; 2: 174. [Crossref]

10. Stone JA, Castillo M, Neelon B, Mukherji SK. Evaluation of CSF leaks: high-resolution CT compared with contrast-enhanced CT and radionuclide cisternography. AJNR Am J Neuroradiol 1999; 20: 706-12. [Crossref]

11. Shetty PG, Shroff MM, Sahani DV, Kirtane MV. Evaluation of high-resolution CT and MR cisternography in the diagnosis of cerebrospinal fluid fistula. AJNR Am J Neuroradiol 1998; 19: 6339. [Crossref]

12. Chan DT, Poon WS, IP CP, Chiu PW, Goh KY. How useful is glucose detection in diagnosing cerebrospinal fluid leak? The rational use of $\mathrm{CT}$ and Beta-2 transferrin assay in detection of cerebrospinal fluid fistula. Asian J Surg 2004; 27: 39-42. [Crossref]

13. Vemuri NV, Karanam LSP, Manchikanti V, Dandamudi S, Puvvada SK, Vemuri VK. Imaging review of cerebrospinal fluid leaks. Indian J Radiol Imaging 2017; 27: 441-6. [Crossref]

14. Eljazzar R, Loewenstern J, Dai JB, Shrivastava RK, Iloreta AM Jr. Detection of cerebrospinal fluid leaks: is there a radiologic standard of care? A systematic review. World Neurosurg 2019; 127: 307-15. [Crossref]

15. Placantonakis DG, Tabaee A, Anand VK, Hiltzik D, Schwartz TH. Safety of low-dose intrathecal fluorescein in endoscopic cranial base surgery. Neurosurgery 2007; 61: 161-5. [Crossref]

16. Demarco RC, Tamashiro E, Valera FC, Anselmo-Lima WT. Use of a hypodense sodium fluorescein solution for the endoscopic repair of rhinogenic cerebrospinal fluid fistulae. Am J Rhinol 2007; 21: 184-6. [Crossref]

17. Qiao X, An H, Liu Y, Liu S, Tang Y, Liang C. [Endoscopic repair of cerebrospinal fluid rhinorrhea]. Lin Chuang Er Bi Yan Hou Ke Za Zhi. 2006; 20: 496-8. [Crossref]

18. Seth R, Rajasekaran K, Benninger MS, Batra PS. The utility of intrathecal fluorescein in cerebrospinal fluid leak repair. Otolaryngol Head Neck Surg 2010; 143: 626-32. [Crossref] 
19. Saafan ME, Ragab SM, Albirmawy OA. Topical intranasal fluorescein: the missing partner in algorithms of cerebrospinal fluid fistula detection. Laryngoscope 2006; 116: 1158-61. [Crossref]

20. Liu HS, Liang H, Wang D, Wang Y, Zheng JP, Wang YP, et al. [Application of topical intranasal fluorescein in endoscopic endonasal repair of cerebrospinal fluid leakage]. Zhonghua Yi Xue Za Zhi 2009; 89: 63-5. [Crossref]

21. Munn Z, Barker TH, Moola S, Tufanaru C, Stern C, McArthur A, et al. Methodological quality of case series studies: an introduction to the JBI critical appraisal tool. JBI Evid Synth 2020; 18: 2127-33. [Crossref]

22. Jones ME, Reino T, Gnoy A, Guillory S, Wackym P, Lawson W. Identification of intranasal cerebrospinal fluid leaks by topical application with fluorescein dye. Am J Rhinol 2000; 14: 93-6. [Crossref]

23. Mantur M, Łukaszewicz-Zając M, Mroczko B, Kułakowska A, Ganslandt O, Kemona H, et al. Cerebrospinal fluid leakage-reliable diagnostic methods. Clin Chim Acta 2011; 412: 837-40. [Crossref]

24. Lipschitz N, Hazenfield JM, Breen JT, Samy RN. Laboratory testing and imaging in the evaluation of cranial cerebrospinal fluid leaks and encephaloceles. Curr Opin Otolaryngol Head Neck Surg 2019; 27: 339-43. [Crossref]
25. Fluorescein uses [Internet]. New York (NY): Generic Drugs. (Cited 25 December 2020). Available at: https://www.ndrugs. $\mathrm{com} /$ ? $\mathrm{s}=$ fluorescein [Crossref]

26. Ren L, Liu Z, Liang M, Wang L, Song X, Wang S. 10 \% fluorescein sodium vs $1 \%$ isosulfan blue in breast sentinel lymph node biopsy. World J Surg Oncol 2016; 14: 280. [Crossref]

27. Shehu BB, Ismail NJ, Hassan I. Management of pneumocephalus in a resource limited environment: review from sub-Saharan Africa. Brain Inj 2007; 21: 1217-23. [Crossref]

28. Safavi A, Safavi AA, Jafari R. An empirical approach to the diagnosis and treatment of cerebrospinal fluid rhinorrhoea: an optimised method for developing countries. Malays J Med Sci 2014; 21: 37-43. [Crossref]

29. Liu HS, Chen YT, Wang D, Liang H, Wang Y, Wang SJ, et al. The use of topical intranasal fluorescein in endoscopic endonasal repair of cerebrospinal fluid rhinorrhea. Surg Neurol 2009; 72: 341-5. [Crossref]

30. Ozturk K, Karabagli H, Bulut S, Egilmez M, Duran M. Is the use of topical fluorescein helpful for management of CSF leakage? Laryngoscope 2012; 122: 1215-18. [Crossref]

31. Eren E, Güvenç G, İşlek A, Arslanoğlu S, Önal K, Yüceer N. Is topical fluorescein that effective in endoscopic CSF leak closure? Eur Arch Otorhinolaryngol 2020; 277: 1073-7. [Crossref] 